\title{
THORACOSCOPIC EN BLOC TOTAL ESOPHAGECTOMY WITH RADICAL MEDIASTINAL LYMPHADENECTOMY
}

Takashi Akaishi, MD

Iwao Kaneda, MD

Norio Higuchi, MD

Yoshiki Kuriya, MD

Jun-ichi Kuramoto, MD

Tsuneo Toyoda, MD

Akio Wakabayashi, MD
Objective: Total esophagectomy with en bloc mediastinal lymphadenectomy for cancer carries a substantial morbidity and mortality rate. To investigate the feasibility of thoracoscopic technique, we carried out an extensive laboratory study. Encouraged by our excellent results, we conducted a clinical trial. Methods: From September 1994 to September 1995, 39 patients thoracic esophageal cancer lesions not invading surrounding organs underwent total esophagectomy with mediastinal lymphadenectomy by means of thoracoscopy. Ages ranged from 47 to 86 years. The procedures were conventional except for the thoracic portion, which was performed as a thoracoscopic procedure with six trocar holes instead of thoracotomy. All harvested lymph nodes were counted for each station. Spirometric data and plethysmographically determined vital capacity were measured before and after operation for all patients. Results: All procedures were accomplished as scheduled, and none was converted to open thoracotomy. The operating time was $200 \pm 41$ minutes (mean \pm standard deviation). Estimated blood loss was $270 \pm 157 \mathrm{ml}$. The harvested lymph nodes numbered $19.7 \pm 11.1$ per patient. Seventeen patients ( $45 \%)$ had positive lymph nodes. There were no in-hospital deaths within 30 days. Twenty-two patients did not require postoperative ventilatory support. Vital capacity decreased to $85 \% \pm 11 \%$ of the preoperative values, and forced expiratory volume in 1 second decreased to $82 \% \pm 16 \%$. Conclusions: Thoracoscopic mediastinal lymphadenectomy is technically feasible, and its completeness is comparable to that of the open technique. The decline in pulmonary function is significantly less than that seen in our previous experience with the open technique. (J Thorac Cardiovasc Surg 1996;112:1533-41)
$E_{p}^{n}$ bloc total esophagectomy with mediastinal and paragastric lymphadenectomy for esophageal carcinoma carries high operative mortality and morbidity rates. ${ }^{1-4}$ It requires laparotomy for the preparation of the stomach or colon to be used as a conduit in addition to retroperitoneal lymphadenec-

From the Second Department of Surgery, Tohoku University School of Medicine, Sendai, Japan, and the Akio Wakabayashi Research Foundation, Irvine, Calif.

Read at the Seventy-sixth Annual Meeting of The American Association for Thoracic Surgery, San Diego, Calif., April 28-May 1, 1996.

Received for publication May 6, 1996; revisions requested May 28, 1996; revisions received August 2, 1996; accepted for publication August 7, 1996.

Address for reprints: Takashi Akaishi, MD, Second Department of Surgery, Tohoku University School of Medicine, 1-1 Seiryo-machi, Aoba-ku, Sendai, Miyagi, Japan 980-77.

Copyright (C) 1996 Mosby-Year Book, Inc.

$0022-5223 / 96 \$ 5.00+0 \quad \mathbf{1 2 / 6 / 7 7 1 8 3}$ tomy, thoracotomy to dissect the esophagus and mediastinal lymph nodes, and cervical incision for gastroesophagostomy or colonoesophagostomy. Respiratory complications from thoracotomy are both the most common and most serious problems. ${ }^{1-4}$ In an effort to minimize morbidity and mortality, Orringer and Sloan ${ }^{5}$ adopted a transhiatal approach. With this technique, the thoracic esophagus is dissected without entering the pleural space. Orringer reported that the incidence of pulmonary complication was significantly reduced and a long-term survival rate was similar to that of radical en bloc esophagectomy. ${ }^{6,7}$ Other investigators ${ }^{8-11}$ concurred with Orringer. The inherent problem with the transhiatal esophagectomy is the inability to visualize the mediastinal structures, including the thoracic aorta and its branches, the venae cavae and the intercostal branches, and the mediastinal lymph nodes. Katariya and colleagues ${ }^{12}$ reviewed 23 published reports on the transhiatal esophagectomy and 


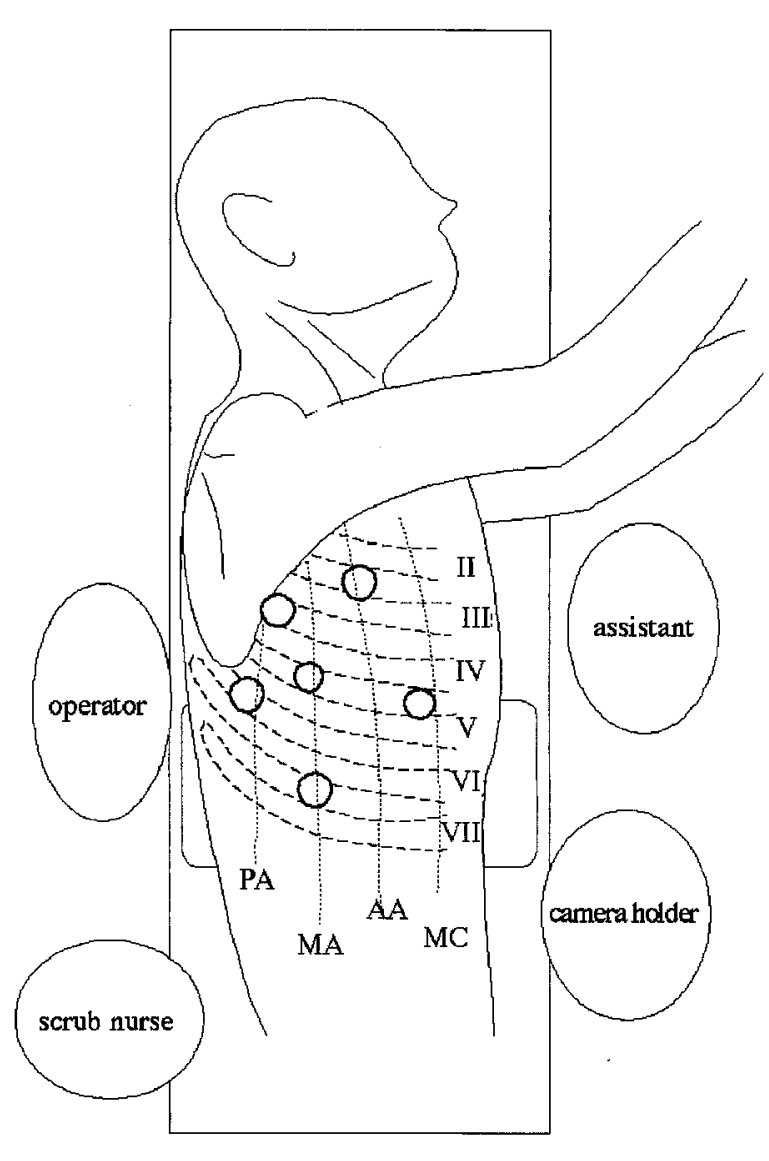

Fig. 1. Trocar sites. Roman numerals indicate ribs. $P A$, Posterior axillar line; $M A$, midaxillary line; $A A$, anterior axillary line; $M C$, midclavicular line.

found that bleeding, tracheal injuries, cardiac arrhythmias, and incidental splenectomy had been reported. It is interesting to note that they found pulmonary complications to be the most common.

With the advent of operative thoracoscopy,${ }^{13}$ it became possible to apply this advanced technique to the resection of the thoracic esophagus. ${ }^{14-18}$ Thoracoscopy has been reported to be associated with significantly less morbidity and provides excellent visualization of the operative field. One question remains, however; can radical mediastinal lymphadenectomy be performed safely by means of thoracoscopy with the same level of completeness as is achieved with a conventional posterolateral thoracotomy? Bessell and coworkers ${ }^{19}$ reported the result of an experimental study on thoracoscopic esophagectomy. They performed thoracoscopic dissection of the thoracic esophagus, laparoscopic dissection of the stomach, and infrathoracic gastroesophagos- tomy with a circular stapler in pigs. Among 10 procedures, one was converted to open thoracotomy, two animals died of anesthetic complications, and one animal bled to death from a hepatic vein injury caused by a liver retractor. Small anastomotic leaks, which were successfully repaired by sutures, were noted in three animals. In their experiments, the mediastinal lymph nodes were not dissected. In light of these results, it appears that the safety and completeness of mediastinal lymphadenectomy by means of thoracoscopy should be studied in the laboratory before attempting clinical trials. In this report, we describe the animal experiments briefly in part I and the early clinical experience with this technique in part II.

\section{Part I: Experimental study}

Objective. The experimental study was conducted with the following objectives: (1) to establish the thoracoscopic technique of en bloc total esophagectomy with mediastinal lymphadenectomy, (2) to investigate the safety of such an operation, and (3) to compare the completeness of mediastinal lymphadenectomy with that of a standard thoracotomy and en bloc mediastinal lymphadenectomy.

Method. Twenty Chester White pigs were divided into two groups of 10 each. In group 1, en bloc total esophagectomy was performed through posterolateral thoracotomy; in group 2, it was performed by thoracoscopy. The guidelines for laboratory animal studies, "Guide for the Care and Use of Laboratory Animals," prepared by the Institute of Laboratory Animal Resources and published by the National Institutes of Health (NIH Publication No. 86-23, revised 1985), were followed, and the protocol was approved by the Animal Research Committee. The animals received intramuscular injections of ketamine hydrochloride and atropine sulfate. An intravenous line was established through an ear vein for hydration and drug administration. Through a tracheostomy, a double-lumen endotracheal tube was inserted and connected to a volume-controlled ventilator-anesthesia machine. Subsequent anesthesia was maintained by halothane (Fluothane; $0.75 \%$ to $1.0 \%$ ). After the cervical esophagus was dissected and transected by a stapler (Linear Cutter; Ethicon Endo-Surgery, Cincinnati, Ohio) through a cervical incision through a right posterolateral thoracotomy, the parietal pleura overlying the vertebral bodies was incised from the first rib to the diaphragm. The azygos arch was doubly ligated with a transfixation technique and divided to obtain good exposure of the left posterior mediastinum. The thoracic duct was divided at the diaphragm. The previously transected cervical esophagus was pulled down into the chest cavity, and gentle traction on it facilitated subsequent dissection of the esophagus and the superior mediastinal soft tissues. The esophagus was separated from the descending thoracic aorta and the membranous part of the trachea. The right bronchial artery was divided at its origin at the intercostal artery, but the left bronchial artery was preserved. The soft tissues were dissected from the pericardium, but the pericardium 


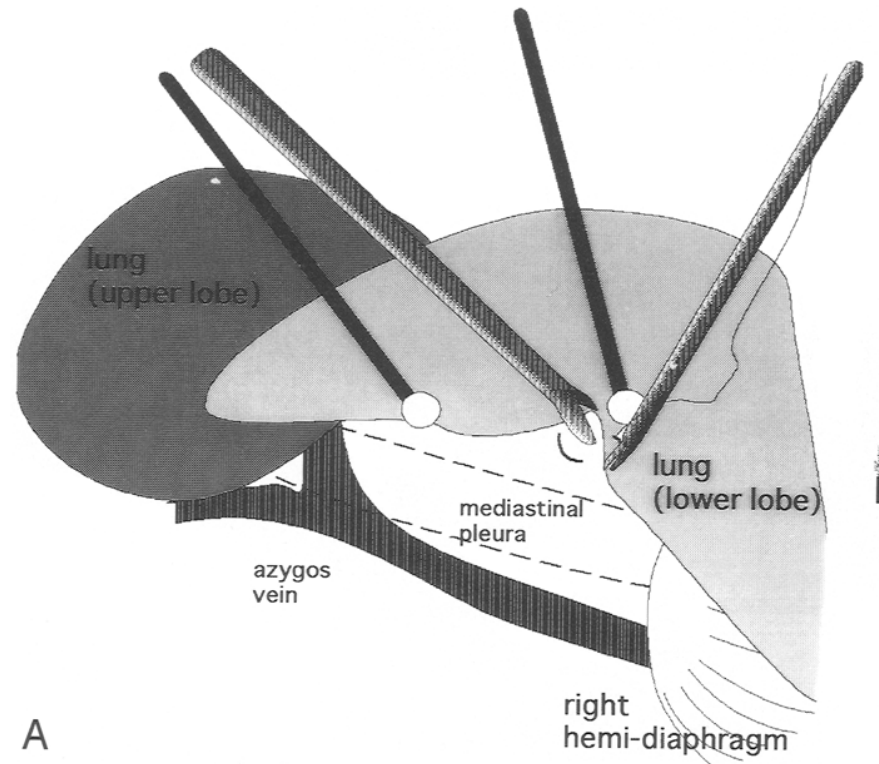

A

hemi-diaphragm

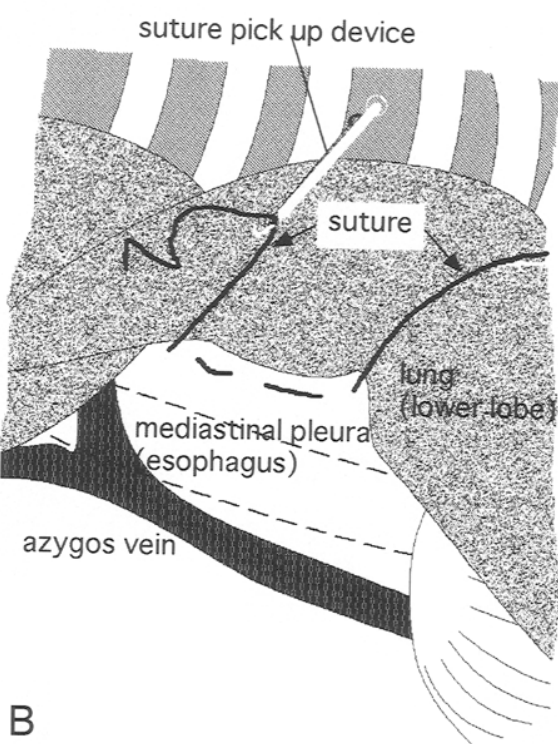

Fig. 2. Marionette technique. A, Step 1. Putting running suture on mediastinal pleura. B, Step 2. Guiding each end of suture and retracting it under mild tension.

was not entered. The soft tissues in the subcarinal region were dissected off the carina and right main bronchus without opening of the left pleura until the left recurrent laryngeal and vagus nerves were identified. The soft tissues in the infraaortic region were dissected off the aortic window, preserving the left recurrent laryngeal and vagus nerves. The right vagus nerve was transected just distal to the takeoffs of the pulmonary branches. After en bloc dissection of the esophagus and surrounding soft tissues, the thoracic esophagus was transected at the level of the diaphragm with a stapler. The esophagus with attached soft tissues was then removed from the chest cavity (en bloc esophagectomy). The remaining mediastinal lymph nodes were sought and removed individually when found. The thoracotomy wound was closed in a routine fashion with two chest tubes, and the animals were weaned from the ventilator.

In group 2, thoracoscopy was performed with one-lung ventilation by means of a standard thoracoscopic technique and with standard equipment. ${ }^{20}$ Five trocars, 10 or $12 \mathrm{~mm}$, were placed in the right lateral portion of the chest. After the posterior parietal pleura was opened widely, the anterior edge of the posterior mediastinal pleura was retracted by two stay sutures to provide good exposure and to keep the lung away from the operative field (referred to as "marionette technique"). The en bloc esophageal dissection with surrounding soft tissues was carried out in a similar fashion to that used in group 1, except that the sequence was reversed in the thoracoscopy group. First, the distal thoracic esophagus was divided at the diaphragm. Traction of the transected esophagus superiorly and anteriorly facilitated the dissection of the soft tissues in the subcarinal and infraaortic regions. The specimen was removed through the cervical incision. The remaining lymph nodes were searched for with the thora-
Table I. Pathologic stages

\begin{tabular}{cc}
\hline Stage & No. of patients \\
\hline I & 9 \\
IIa & 9 \\
IIb & 3 \\
III* & 12 \\
IV $\dagger$ & 6 \\
\hline
\end{tabular}

*All T3, N1, M0

$\dagger$ All classified M1 because of positive lymph node beyond the regional areas.

coscope and removed individually if found. After two chest tubes were placed through trocar holes, other trocar holes were closed and the animal was weaned from the ventilator. In both groups, the animals were considered survivors when they resumed normal spontaneous breathing and their vital signs remained stable for at least an hour. Operating time and estimated blood loss were recorded in each experiment. On completion of the experiment, the animals were killed by intravenous barbiturate injection and subjected to autopsy. Medial portions of the ribs were removed to obtain a wide exposure, and the bilateral mediastinum was thoroughly inspected. If any residual mediastinal lymph nodes were found, they were removed and labeled according to the station. The numbers of lymph nodes harvested during the operation and of those overlooked were recorded. Student's $t$ test was used to compare two groups and a $p$ value of less than 0.05 was considered significant.

Results. All animals tolerated the procedures. The operating time of group 1 was $108 \pm 10$ minutes, and that of group 2 was $169 \pm 50$ minutes (mean \pm standard deviation). The difference was significant. The estimated 


\section{\# total number of harvested lymph nodes \\ $\$$ frequency of metastasis per number of cases \\ @ frequency of metastasis per number of nodes}

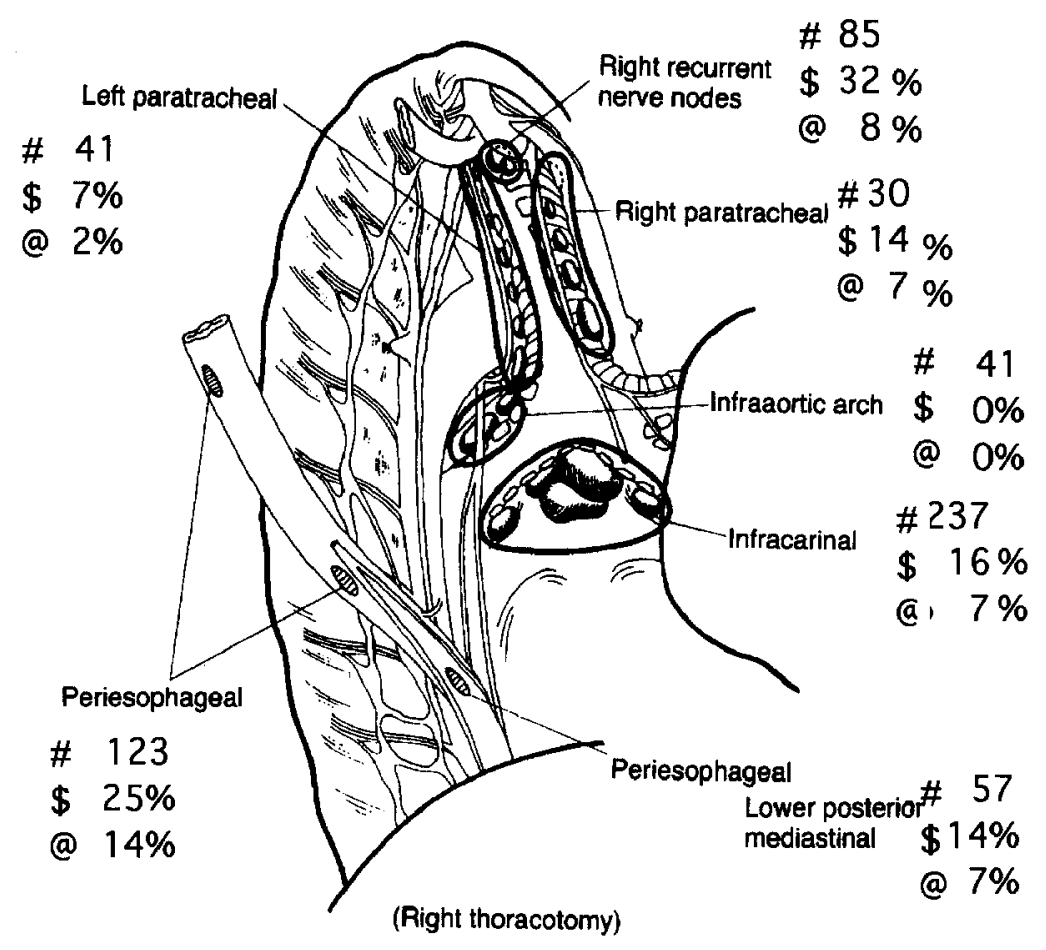

Fig. 3. Distribution of harvested mediastinal lymph nodes and incidence of metastasis. (Adapted from Kakegawa T, Hujita H, Yamana H. Illustrations of surgery for carcinoma in the thoracic esophagus. In: Sato T, Iizuka T, editors. Color atlas of surgical anatomy for esophageal cancer. Tokyo: Springer-Verlag, 1992:91-116. Used with permission.)

blood loss of group 1 was $52 \pm 36 \mathrm{ml}$; that of group 2 was $67 \pm 46 \mathrm{ml}$, and the difference was not significant. The total numbers of the excised lymph nodes were 112 in group 1 and 125 in group 2; the difference between the two groups was insignificant. The right paratracheal lymph nodes removed en bloc by thoracoscopy were significantly more numerous than in the thoracotomy group, but there were no significant differences in other stations.

Conclusions. Thoracoscopic en bloc esophagectomy with mediastinal lymphadenectomy is technically feasible, is safe, and provides satisfactory lymph node dissection, comparable to that of an open technique.

\section{Part II: Early clinical experience}

Patients and methods. After the protocol was approved by the Review Board of Progressive Clinical Practice in Tohoku University School of Medicine, this clinical investigation was initiated. From September 1994 to September 1995, 46 patients with carcinoma of the esophagus were evaluated. Four were found to have carcinoma in situ and were treated by endoscopic resection of mucosa. In three, the carcinomas were invading the descending thoracic aorta or tracheobronchial tree and were treated by chemotherapy, radiation, or both. The remaining 39 patients ( 32 male and seven female) were selected for this study. The average age was 67 years, ranging from 47 to 86 years. The distribution of cancers was as follows: one upper third of the esophagus, 20 middle third, and 18 distal third. All cancers were squamous cell.

The thoracoscopic technique used in patients was similar to that described in part $I$, except the tracheostomy was not necessary and the sequence of the operation was modified to thoracoscopy followed by laparotomy and the cervical incision. First, patients were positioned on an operating table in a left decubitus position. Six trocars, through which all intrathoracic maneuvers were carried out, were placed as shown in Fig. 1. No access or utility thoracotomy was created. Dissection of the soft tissues was performed with the endoscopic instruments, including $10 \mathrm{~mm}$ scissors, $5 \mathrm{~mm}$ grasping forceps, and cherry dissector. During dissection, care was taken not to touch lymph nodes. The entire posterior mediastinal pleura was opened widely, and the anterior edge of the pleura was 
Table II. Postoperative complications

\begin{tabular}{lc}
\hline \multicolumn{1}{c}{ Complication } & No. of patients \\
\hline Pulmonary & \\
Pneumonia & 3 \\
Atelectasis & 5 \\
Air leak & 1 \\
Pleural cavity & \\
$\quad$ Effusion & \\
Chylothorax & 1 \\
Cardiac & \\
$\quad$ Paroxysmal supraventricular tachycardia & 2 \\
Anastomotic leak (minor) & 2 \\
Recurrent nerve paresis & \\
Right & 5 \\
Left* & 1 \\
Bilateral & 1 \\
\hline
\end{tabular}

${ }^{*}$ Continued longer than 6 months.

retracted by two stay sutures as described in part I (marionette technique, Fig. 2). This maneuver was effective in holding the lung away and in providing an excellent field of vision. The azygos arch was divided by means of an endoscopic stapler with vascular cartridges, facilitating dissection of the left side of the posterior mediastinum. The descending thoracic aorta was freed anteriorly, separating the esophagus; the thoracic duct was spared. If enlarged lymph nodes could not be separated from the thoracic duct, it was doubly ligated and removed with the lymph nodes. The esophageal hiatus was dissected, and the anterior esophageal wall was freed from the pericardium. The gastrointestinal branch of the right vagus nerve was divided beneath the right main bronchus, preserving the pulmonary branches. Subcarinal lymph nodes attached to the esophagus were dissected from the carina. The left bronchial artery was preserved to maintain blood supply to the tracheobronchial tree. The cranial one third of the esophagus was separated from the membranous part of the trachea. The right recurrent laryngeal nerve lymph nodes were dissected at the level of the right subclavian artery, with extreme caution taken to avoid injury to the nerve. The path of the left recurrent laryngeal nerve was identified by pushing the trachea anteriorly, and the lymph nodes around the nerve were dissected. After the esophagus was completely dissected from the mediastinum, it was divided at the cancer-free point, using an endoscopic stapler (Endopath LC, Ethicon Endo-Surgery, Cincinnati, Ohio). After the esophagus was divided, both ends were anchored with heavy sutures for the subsequent pull-through procedure. The pleural traction sutures were removed, and two chest tubes were placed through trocar holes. Other trocar holes were closed in layers.

The patient was turned to a supine position, and a midline upper laparotomy was made. Simultaneously, a cervical incision was made on either the left or the right side to expose and dissect the cervical esophagus. The remnant of the esophagus was pulled down into the abdomen to facilitate the dissection of the paragastric lymph nodes. The esophagus and cardia were excised with attached lymph nodes. The left gastric artery was divided

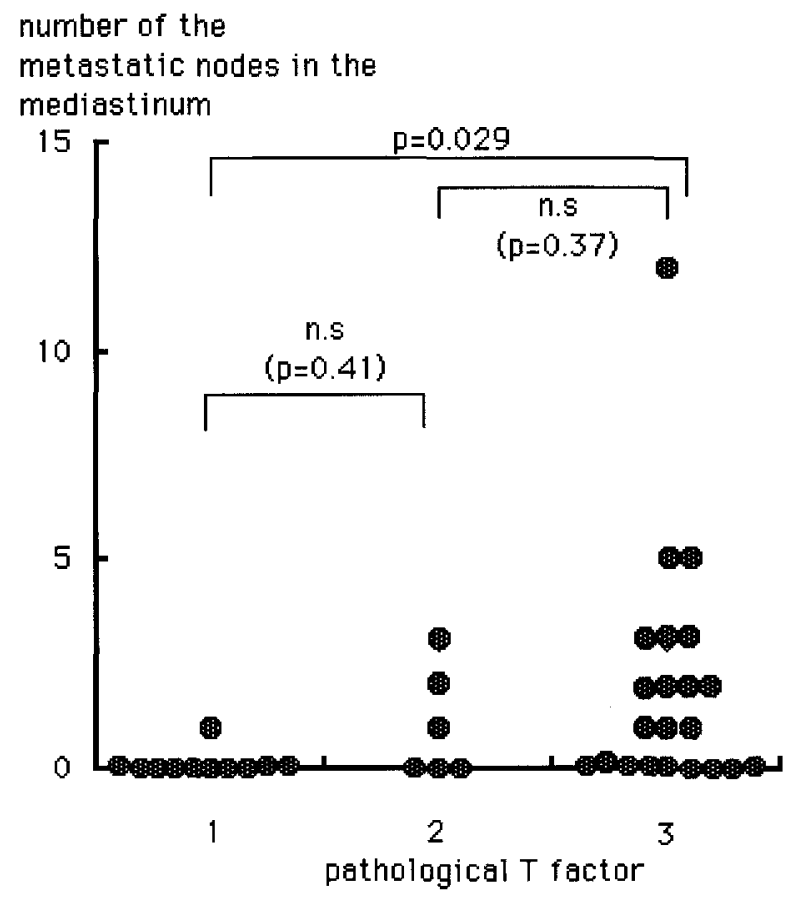

Fig. 4. Correlation between wall penetration of tumor (pathologic $\mathrm{T}$ factor) and incidence of mediastinal lymph node metastasis. $n s$, Not significant.

at its origin. The short gastric and left gastroepiploic vessels were divided and the right gastric and right gastroepiploic arteries were left intact. With staplers (LC-50, Ethicon Endo-Surgery), the greater curvature side of the stomach was fashioned to form a gastric tube. Seromuscular sutures were added to reinforce the staple line. The pyloric sphincter was dilated by invaginating the duodenum into the pylorus with a thumb. The gastric tube was fixed to the previously placed suture on the esophagus. When the distal cervical esophagus was pulled, the thoracic esophagus and the anchored gastric tube were brought out to the neck together. Cervical paratracheal lymph nodes were dissected if found. The anastomosis between the cervical esophagus and the gastric tube was made in two layers with interrupted 4-0 PDS II sutures (Ethicon, Inc., Somerville, N.J.) for the muscular layer and 4-0 silk for the seromuscular layer. After the anastomosis was completed, the gastric tube was brought back into the posterior mediastinum by pulling the gastric tube from the abdomen, positioning the anastomosis in the posterior mediastinum. An epidural catheter was placed before induction of anesthesia to facilitate postoperative pain management.

The intraoperative data and preoperative and postoperative spirometric measures were collected and analyzed with a standard statistical method. The data included operating time (in minutes), estimated blood loss (in milliliters), the incidence and duration of postoperative ventilator support, any postoperative complications, pathologic staging (International Union Against Cancer $\left[\mathrm{UICC}^{21}\right.$ ) of the surgical specimens, the depth of the 
Vital Capacity

Change Ratio

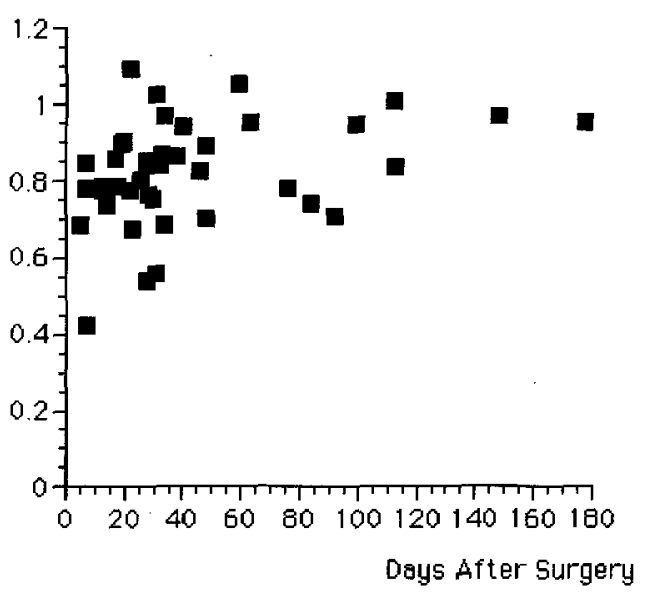

FEY 1

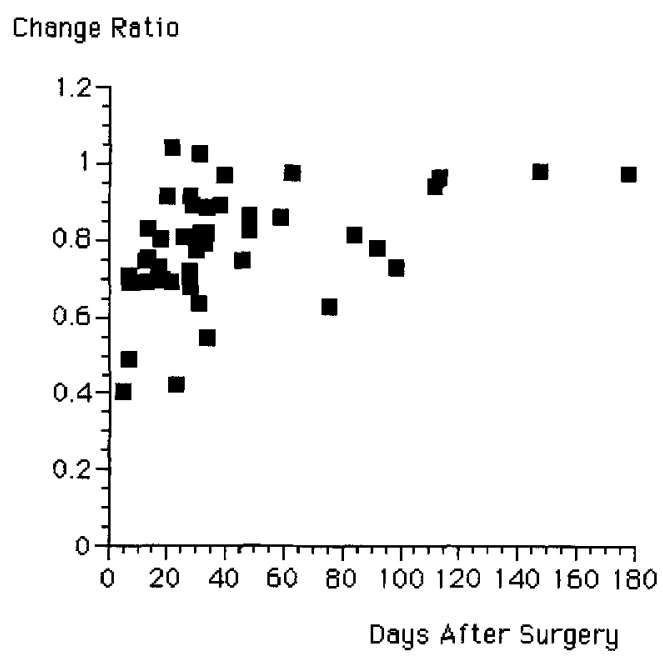

\section{Postoperative Pulmonary Functions}

Fig. 5. Postoperative changes in spirometric data. FEV1, Forced expiratory volume in 1 second.

invasion of the primary tumors, and the presence of malignant cells in the harvested lymph nodes for each station (International Society for Diseases of the Esophagus $[\mathrm{ISDE}]^{22,23}$ ). Preoperative and early postoperative spirometric measurements, including vital capacity and forced expiratory volume in 1 second, were obtained whenever the patients tolerated the tests. Postoperative values were expressed as percentage of preoperative value.

Results. All patients tolerated the procedure well, and the thoracoscopic esophagectomy was successfully completed in all cases. No procedures were converted to open thoracotomy. The operating time for thoracoscopy was $200 \pm 41$ minutes and the total operating time was $448 \pm$ 67 minutes. The estimated blood loss for thoracoscopy was $270 \pm 157 \mathrm{ml}$, and the total estimated blood loss was $767 \pm 783 \mathrm{ml}$. The number of harvested lymph nodes per patient were as follows: mediastinal, $19.7 \pm 11.1$; and paragastric, $12.5 \pm 9.0$. Cervical lymph nodes were dissected in 13 patients. The pathologic stages are shown in Table I. The distribution of the harvested mediastinal lymph nodes and the incidence of metastasis are shown in Fig. 3. There was a significant correlation between the depth of the tumor and the incidence of mediastinal lymph node metastasis (Fig. 4). Twenty-two patients were extubated in the operating room, and 14 were weaned from the ventilator within 2 days. Three required ventilatory support for 5 to 30 days. These results were significantly better than our previous experience with conventional open technique. ${ }^{24}$ No operative deaths occurred.

Postoperative complications are listed in Table II. Atelectasis resolved after bronchoscopic lavage and physiotherapy. Hoarseness developed in seven patients $(18 \%)$. All but one of these patients regained voice within 6 months. One patient required a polytetrafluoroethylene (Teflon) implant to restore voice.

Fig. 5 shows postoperative changes in spirometric values. At 1 month after operation, vital capacity fell to $83 \%$ and forced expiratory volume in 1 second fell to $85 \%$, but both recovered to the preoperative levels in 3 months.

\section{Discussion}

Our experimental study indicated that thoracoscopic en bloc total esophagectomy with mediastinal lymphadenectomy is safe, and it is as effective as the same procedure performed through a posterolateral thoracotomy. It was interesting that more lymph nodes were removed from the right paratracheal station en bloc by thoracoscopy than through a thoracotomy, which may be the result of better visualization. Collard and associates ${ }^{16}$ reported 12 thoracoscopic esophagectomies, two of which were converted to open procedures (17\%). Of their four patients who underwent extensive en bloc esophageal resection, two required blood transfusions. The number of lymph nodes removed ranged from 21 to 51. Pneumonia developed in two patients. Collard and associates ${ }^{16}$ concluded that thoracoscopic esophagectomy was feasible but did not completely prevent respiratory complications. In our experience, it took several months of experiments before we felt comfortable with the thoracoscopic technique in animals, and we practiced the technique in 
our laboratory for more than 18 months before we attempted the first clinical case. We believe that such an extensive laboratory experience is crucial to achieving a high success rate in a clinical setting, a belief supported by the fact that no thoracoscopic procedure was converted to thoracotomy. Mediastinal lymphadenectomy was achieved without difficulty in all cases, and no major complications occurred. Our previous experience indicated that en bloc esophagectomy through thoracotomy was associated with a high incidence (33\%) of pulmonary complications. ${ }^{24}$ Although these still occurred with the thoracoscopic approach, their incidence was significantly lower $(20 \%)$.

A relatively high incidence of hoarseness has been reported after esophagectomy, ${ }^{6,25}$ but this hoarseness is usually transient. In our previous experience with radical esophagectomy, transient hoarseness developed in $80 \%$ of the patients. ${ }^{24}$ In this series, transient hoarseness developed in six cases, with one case of permanent left recurrent laryngeal nerve paralysis. We believe that the improved result can be attributed to the magnification and excellent visualization provided by thoracoscopy.

The initial pulmonary function test results of our patients indicated that their function was well preserved, despite upper abdominal laparotomy. If the abdominal procedure is done with laparoscopy, the pulmonary function may be even better preserved. Many patients with esophageal cancers have poor pulmonary reserve and are not considered candidates for thoracotomy. These patients have been treated by external radiation, with no long-term survivors. By means of this thoracoscopic en bloc esophagectomy, we may be able to treat these patients surgically with improved results. A longterm follow-up study is necessary to see whether thoracoscopic en bloc esophagectomy would offer a long-term survival rate equivalent or superior to that of the conventional open technique.

\section{Conclusions}

The en bloc total esophagectomy by means of thoracoscopy is safe. The completeness of mediastinal lymph node dissection is equivalent to that of the conventional open technique. Despite excellent visualization of the recurrent laryngeal nerve, postoperative hoarseness developed in $18 \%$ of patients, with one case permanent. At 1 month after operation, pulmonary function had returned to $85 \%$ of the preoperative level. At 3 months, it was back to the preoperative level. The incidence of pulmonary complication is reduced with this technique. Patients with restricted pulmonary reserve, who would not tolerate conventional en bloc total esophagectomy through an open thoracotomy, may tolerate the thoracoscopic approach. A long-term survival study is required to evaluate this technique.

We express our gratitude to the following students of the School of Biological Science at the University of California Irvine: Norihisa Sato, Martin E. Dahl, Toshiyuki Hori, Fariba Derakhshanfar, Sumina Fukami, and Yukiaki Miyata, for their participation in the experiments as a part of their extended experiment course. The following affiliated hospitals participated in this clinical study: Ishinomaki Red Cross Hospital, Sakata City Hospital, Iwate Prefectural Iwai Hospital, Furukawa City Hospital, Kamaishi City Hospital, and Hachinohe City Hospital.

\section{REFERENCES}

1. Logan A. The surgical treatment of carcinoma of the esophagus and cardia. J Thorac Cardiovasc Surg 1963;46:150-61.

2. Isono K, Onda S, Ishikawa T, Sato H, Nakayama K. Studies of the causes of deaths from esophageal carcinoma. Cancer 1982;49:2173-9.

3. Skinner DB. En bloc resection for neoplasms of the esophagus and cardia. J Thorac Cardiovasc Surg 1983;85:59-71.

4. Matory YL, Burt N. Esophagogastrectomy: reoperation for complications. J Surg Oncology 1993;54:29-33.

5. Orringer MB, Sloan H. Esophagectomy without thoracotomy. I Thorac Cardiovasc Surg 1978;76:643-54.

6. Orringer MB, Orringer JS. Esophagectomy without thoracotomy: a dangerous operation? J Thorac Cardiovasc Surg 1983;85:72-80.

7. Orringer MB, Marshall B, Stirling MC. Transhiatal esphagectomy for benign and malignant disease. J Thorac Cardiovasc Surg 1993;105:265-76.

8. Gertsch P, Vauthey JN, Lustenberger AA, Fiedlander-Klar H. Long-term results of transhiatal esophagectomy for esophageal carcinoma: a multivariate analysis of prognostic factors. Cancer 1993;72:2312-9.

9. Pac M, Baoglu A, Kocac H, Yekeler I, Yediyildiz S, Aydin NE, et al. Transhiatal versus transthoracic esophagectomy for esophageal cancer. J Thorac Cardiovasc Surg 1993;106:205-9.

10. Bolton JS, Ochsner JL, Abdoh AA. Surgical management of esophageal cancer: a decade of change. Ann Surg 1994;219: $475-80$.

11. Millikan KW, Silverstein J, Hart V, Blair K, Bines S, Roberts J, et al. A 15-year review of esophagectomy for carcinoma of the esophagus and cardia. Arch Surg 1995;130:617-24.

12. Katariya K, Harvey JC, Pina E, Beattie EJ. Complications of transhiatal esophagectomy. J Surg Oncol 1994;57:157-63.

13. Wakabayashi A. Expanded applications of diagnostic and therapeutic thoracoscopy. J Thorac Cardiovasc Surg 1991; 102:721-3.

14. Cuschieri A, Shimi S, Banting S. Endoscopic oesophagectomy through a right thoracoscopic approach. J R Coll Surg Edinb 1992;37:7-11.

15. Gossot D, Fourquier P, Celerier M. Thoracoscopic esoph- 
agectomy: technique and initial results. Ann Thorac Surg 1993;56:667-70.

16. Collard JM, Lengele B, Otte JB, Kestens PJ. En bloc and standard esophagectomies by thoracoscopy. Ann Thorac Surg 1993;56:675-9.

17. Pellegrini CA. Esophageal surgery by the thoracoscopic approach. Semin Thorac Cardiovasc Surg 1993;5:305-9.

18. McAnena OJ, Roers J, Willaims NS. Right thoracoscopically assisted oesophagectomy for cancer. Br J Surg 1994;81:236-8.

19. Bessell JR, Maddern GJ, Manncke K, Ludbrook G, Jamiesen GG. Combined thoracoscopic and laparoscopic oesophagectomy and oesophagogastric reconstruction. Endoscopic Surg Allied Technol 1994;2:32-6.

20. Wakabayashi A. Laser treatment of pseudopneumocysts of the lung. In: Gossot D, Kleinmann P, Levi JF, editors. Surgical thoracoscopy. Paris: Springer-Verlag, 1992:45-55.

21. SpiessI B, Beahrs OH, Hermanek P, Hutter PVP, Scheibe O, Sobin LH, et al, editors. International Union Against Cancer TNM classification on malignant tumors, 4th ed. Berlin: Springer-Verlag, 1989:62-3.

22. Sato T, Sakamoto K. Illustrations and photographs of surgical esophageal anatomy specially prepared for lymph node dissection. In: Sato T, Iizuka T, editors. Color atlas of surgical anatomy for esophageal cancer. Tokyo: Springer-Verlag, 1992:25-90

23. Kakegawa T, Hujita H, Ymana, H. Illustrations of surgery for carcinoma in the thoracic esophagus. In: Sato $T$, Iizuka $T$, editors. Color atlas of surgical anatomy for esophageal cancer. Tokyo: Springer-Verlag, 1992:91-116.

24. Nishihira T, Mori S, Hirayama K. Extensive lymph node dissection for thoracic esophageal carcinoma. Dis Esophagus 1992;5:79-89.

25. Johnson PR, Kenegonanker GS, Bates T. Indirect laryngoscopic evaluation of vocal cord function in patients undergoing transhiatal esophagectomy. J Am Coll Surg 1994;178: $605-8$.

\section{Discussion}

Dr. David B. Skinner (New York, N.Y.). Thank you for a fine paper, and the manuscript read equally well. I wanted to make just a few comments. You used the terms extended lymphadenectomy and en bloc. Actually, those are somewhat different concepts, and you certainly did a fine extended lymphadenectomy. The en bloc concept that we have been discussing through the years involves removing all the adjacent fatty tissue and pleural surfaces as well as lymph nodes around the esophagus, so that one does not actually dissect on the esophagus. Just for the sake of accuracy, I think we should make that distinction. There is no evidence at this point that extended lymphadenectomy or en bloc resection differ with respect to outcome, but both provide significantly better results, statistically significant now in seven or eight series, for stage I and stage II esophageal cancer. At the Consensus Conference in Europe last summer, there was uniform agreement around the world that for early-stage cancer one should do an extended operation of one type or the other, and we do not know whether there is a real difference between en bloc and the radical lymphadenectomy that you showed today.

Your results are excellent, and there have never been results this good reported for thoracoscopic esophagec- tomy. I think the reason for that is your pig experience; you were experienced with how to do the operation in animals before you took it to human beings. I would recommend that strongly to all of us who would have an interest in proceeding with this technique. Clearly, you are seeing things better and doing a safer operation, I would judge, this way than by doing it transhiatally with blunt dissection. This does recommend it to people who use the transhiatal approach for esophagectomy.

I have two questions. First, in the pig series you did a more extensive resection in that you took the thoracic duct and more of the posterior tissues behind the esophagus than did you in the human patients. Was there a particular reason that you changed the technique to one slightly less extensive in the human patients? Second, did you have cases of a large middle third tumor? If so, did you encounter difficulties with that particular kind of tumor? Those are the ones that cause the trouble in the transhiatal blunt dissection.

Dr. Akaishi. I would like to answer your first question as to the thoracic duct. When the tumors were substantial in size, we resected the thoracic duct in the clinical cases; when the tumors were not so big, we preserved the thoracic duct. Thank you for pointing out the usages of $e n$ bloc and extended lymphadenectomy. I think that the advantage of that technique is to preserve what should be preserved and resect what should be resected. It was not rare for me to find big tumors in the middle of the esophagus, and all of those tumors were resectable in my series. Please note that we preoperatively excluded the patients with tumors appearing to invade surrounding organs.

In answer to your second question, if I have big tumor at the middle of the esophagus and if someone considers it inconvenient to do transhiatal esophagectomy, I think the technique I described is worth trying.

Dr. Mark Krasna (Baltimore, Md.). I also would like to congratulate you on an elegant study. I think that it is important to note that a couple of years ago this was attempted in Europe by Collard and associates, ${ }^{16}$ and they actually abandoned it because the operative time was so long. I think that you have achieved an important technical feat in shortening the operating time. I agree that this is probably a result of your animal experience. We all owe a debt to Skinner, Akiyama, Ellis, and recently Steup and Lerut from Belgium, for showing the prognostic importance of lymph node staging. Whether someone's philosophy involves radical, en bloc, or extensive lymphadenectomy or not, I think it is clear that getting lymph node staging before resection will allow us to decide what type of operation to use, or whether patients should receive combined-modality therapy. Thoracoscopic lymph node sampling, as you have shown, or extensive sampling does, I think, hold promise for the future.

I have one question. Can you please tell us where the primary tumors were- upper, middle and lower thirdand what numbers of lymph nodes were from each area? According to those numbers, can you explain why you think there were so few patients who had positive abdominal lymph nodes? Akiyama and many other investigators have noticed as many as $30 \%$ and even $50 \%$ positive.

Dr. Akaishi. In answer to your first question, according 
to my experience, extent of the lymph node dissection would be decided by the site of the tumor. We had only about two or three cases of upper third tumor, and the remaining were middle or lower third. Further, the operative extent must be decided not by thoracotomy or by thoracoscopy, but by which part the tumor resides in. For example, for the upper third I would excise mainly the lymph nodes in the neck and upper mediastinum, but in the middle third, the lymphatic metastasis diverges at any place in the neck, mediastinum, or abdomen. So we should do extensive three-field lymphadenectomy. In the case of a tumor located in the lower third, abdominal node dissection would play the main role. Again, it is not decided by the method of operation but the site of the tumor.

Dr. Antoon Lerut (Leuven, Belgium). I congratulate you on a fine effort, and also stress the importance of first going to the animal laboratory and then to the human patients, and not vice versa.

I have a couple of questions concerning the technique. You have been focusing on the lymphadenectomy, but you have not been elaborating on the completeness of the primary resection of the tumor, the $\mathrm{T}$ factor, and the $\mathrm{R} 0$ status. I noticed that you had a number of $\mathrm{T} 3$ and $\mathrm{T} 4$ tumors. Did you achieve an R0 situation in every patient?
How many patients had a lateral positive section plane, which is also an important prognostic factor? Second, the lymph node dissection, at least in my experience with thoracoscopy, often results in fragmentation of lymph nodes. You said that the number of lymph nodes was a mean of 19. Are those complete lymph nodes, or fragments of lymph nodes? I think that is an important issue. Finally, we are all concerned about trocar inoculation; there have been some reports on that, probably because of increased manipulation of the tumors. Of course your experience is recent, but did you see any early recurrence in the mediastinum or any trocar inoculation?

Dr. Akaishi. To your first question, we had no palliative cases among our 39 patients in this series, but we have now seen 77 cases. Among the 77 patients, we have one in whom tumor remained at the left main bronchus. In choosing this operative technique, we exclude meticulously if the computed tomographic scan tells us that tumor may invade surrounding organs. Unfortunately, we have one palliative case in which the tumor remained in the left bronchus.

With respect to fragmentation of the lymph node, I agree that I might divide lymph node one to two, but I did not find a better way to assay or determine surgical fragmentation. 